\title{
NECESSARY AND SUFFICIENT CONDITIONS FOR CODIMENSION- $k$ MAPS TO BE APPROXIMATE FIBRATIONS
}

\author{
YOUNG Ho IM
}

\begin{abstract}
Let $N$ be a closed $n$-manifold with residually finite, torsion free $\pi_{1}(N)$ and finite $H_{1}(N)$. Suppose that $\pi_{k}(N)=0$ for $1<k<n-1$. We show that $N$ is a codimension- $n$ PL fibrator if and only if $N$ does not cover itself regularly and cyclically up to homotopy type, provided $\pi_{1}(N)$ satisfies a certain condition.
\end{abstract}

\section{Introduction}

Approximate fibrations represent an extremely important class of maps, due to the presence of various sequences presenting computable homotopical and homological relationships involving the domain, image and typical fiber. (See $[2,3,4]$ for the definition and usefulness of approximate fibrations.) The advantage of this notion is that on one hand there exists an exact homotopy sequence but on the other hand there are more such approximate fibrations available.

This paper seeks to identify homotopy types by means of which a proper map defined on an arbitrary manifold of a given dimension can be quickly recognized as an approximate fibration, simply because all point preimages have the specified homotopy type. More precisely, the goal is to present closed $n$-manifolds $N$ which force proper maps $p: M \rightarrow B$ to be approximate fibrations, when $M$ is an $(n+k)$-manifold and each $p^{-1}(b)$ has the homotopy type (or, more generally, the shape) of $N$. Such a manifold $N$ is called a codimension- $k$ fibrator.

Received February 28, 2002.

2000 Mathematics Subject Classification: Primary 57N15, 55M25; Secondary $57 \mathrm{M} 10,54 \mathrm{~B} 15$.

Key words and phrases: residually finite group; hopfian manifold; approximate fibration.

This work was supported by Korea Research Foundation Grant (KRF-2000-041D00023). 
To explain what all this means and to limit the focus somewhat, we begin by presenting the notation and fundamental terminology to be employed throughout: $M$ is a connected $(n+k)$-manifold and $p: M \rightarrow$ $B$ is a proper map of $M$ to a space $B$ such that each $p^{-1}(b)$ has the homotopy type of a closed, connected $n$-manifold. Such a map $p$ will be called a codimension- $k$ map. When $N$ is a fixed PL $n$-manifold, $M$ is a $\mathrm{PL}$ manifold, $B$ is a polyhedron, and $p: M \rightarrow B$ is a $\mathrm{PL}$ map, then $p$ is said to be $N$-like if each $p^{-1}(b)$ collapses to an $n$-complex homotopy equivalent to $N$. We call $N$ a codimension-k PL fibrator if, for every PL $(n+k)$-manifold $M$ and $N$-like PL map $p: M \rightarrow B, p$ is an approximate fibration. Finally, if $N$ is a codimension- $k$ PL fibrator for all $k>0$, we simply call $N$ a $P L$ fibrator. Many closed manifolds are known to be codimension- $k$ PL fibrators $[8,9,10]$. Surprisingly few nonfibrators are known so far. The best well-known nonfibrators are closed manifolds that cyclically covers itself nontrivially, such as $S^{1} \times S^{1}$ and $\mathbb{R} P^{n} \# \mathbb{R} P^{n}$ [5, Theorem 4.2]. Daverman gives some other kind of nonfibrator, which is $L(p, q) \times S^{3}$. None of them gives necessary and sufficient conditions for closed manifolds to fibrators in general cases. Recently, Im and Kim $[13,14]$ gave some necessary and sufficient conditions for closed manifolds to be codimension-2 fibrators in several cases.

In this paper, we show that as our main result, $N$ is a codimension$n(n \geq 2)$ PL fibrator if and only if $N$ does not cover itself regularly and cyclically up to homotopy type, provided $\pi_{1}(N)$ satisfies certain condition, when $N$ is a closed $n$-manifold with residually finite, torsion free $\pi_{1}(N)$, finite $H_{1}(N)$, and $\pi_{k}(N)=0$ for $1<k<n-1$.

\section{Definitions and preliminaries}

Throughout this paper, the symbols $\simeq$ and $\cong$ denote a homotopy equivalence and an isomorphism, respectively. All manifolds are understood to be finite dimensional, connected and orientable.

A surjective map $p: M \rightarrow B$ between locally compact ANRs is an approximate fibration, a concept introduced and studied by Coram and Duvall $[2,3]$, if it has the following approximate homotopy lifting property: given an open cover $\Omega$ of $B$, an arbitrary space $X$, and maps $f: X \rightarrow M$ and $F: X \times I \rightarrow B$ satisfying $p \circ f=F_{0}$, there exists a map $F^{\prime}: X \times I \rightarrow M$ such that $F_{0}^{\prime}=f$ and $p \circ F^{\prime}$ is $\Omega$-close to $F$. The latter means that to each $x \in X$ corresponds $U_{x} \in \Omega$ with $\left\{p F^{\prime}(x), F(x)\right\} \subset U_{x}$. 
Say that a group $\Gamma$ is sparsely Abelian if it contains no nontrivial normal subgroup $A$ such that $\Gamma / A$ is isomorphic to a normal subgroup of $\Gamma$. Groups $\Gamma$ that are both sparsely Abelian and normally cohopfian have the useful feature that every homomorphism $\Gamma \rightarrow \Gamma$ with, at worst, Abelian kernel necessarily is an automorphism. For brevity, a group $\Gamma$ which is both normally cohopfian and sparsely Abelian will be said to have Property NCSA.

A group $\Gamma$ is said to be: hopfian if each epimorphism $\Gamma \rightarrow \Gamma$ is an isomorphism; cohopfian if each monomorphism $\Gamma \rightarrow \Gamma$ is an isomorphism; and normally cohopfian if each monomorphism $\Gamma \rightarrow \Gamma$ with image a normal subgroup of $\Gamma$ is an isomorphism.

Let $N$ and $N^{\prime}$ be (not necessarily closed) $n$-manifolds and $f: N \longrightarrow$ $N^{\prime}$ be a map. Denote the $k$ th cohomology group of $N$ with $\mathbb{G}$-coefficients and compact supports by $H_{C}^{k}(N ; \mathbb{G})$. The degree of $f$ is the nonnegative integer $d$ such that the induced endomorphism $f^{*}: H_{C}^{n}(N ; \mathbb{Z}) \cong \mathbb{Z} \longrightarrow$ $H_{C}^{n}\left(N^{\prime} ; \mathbb{Z}\right) \cong \mathbb{Z}$ amounts to multiplication by $d$, up to sign. A closed, orientable manifold $N$ is said to be hopfian if every degree 1 map $N \rightarrow$ $N$ which induces an isomorphism at the fundamental group level is a homotopy equivalence. As a result, when $\pi_{1}(N)$ is a hopfian group, $N$ is a hopfian manifold if and only if all degree 1 maps $N \rightarrow N$ are homotopy equivalences. According to Hausmann [11, Proposition 1], every closed orientable manifold of dimension at most 4 is Hopfian.

Suppose that $N$ is a closed $n$-manifold and a proper map $p: M \longrightarrow B$ is $N$-like. Let $G$ be the set of all fibers, i.e., $G=\left\{p^{-1}(b): b \in B\right\}$. Put $C=\left\{p(g) \in B: g \in G\right.$ and there exist a neighborhood $U_{g}$ of $g$ in $M$ and a retraction $R_{g}: U_{g} \longrightarrow g$ such that $R_{g} \mid g^{\prime}: g^{\prime} \longrightarrow g$ is a degree one map for all $g^{\prime} \in G$ with $g^{\prime} \in G$ in $U_{g}$. Call $C$ the continuity set of $p$. D. Coram and P. Duvall showed that $C$ is a dense, open subset of $B$ $[4]$.

A codimension- $k$ map $p: M^{n+k} \rightarrow B$ is said to have Property $\mathcal{R}_{i}^{\cong}$ if for each $x \in B$, a retraction $\mathcal{R}: p^{-1}(U) \rightarrow p^{-1}(x)$ defined on some open neighborhood $U$ of $x$ in $B$ induces an isomorphism $\left(\mathcal{R} \mid p^{-1}(y)\right)_{*}$ : $H_{i}\left(p^{-1}(y)\right) \rightarrow H_{i}\left(p^{-1}(x)\right)$ for all $y \in U$. The continuity set of $p$ consists of all $x \in B$ equipped with such a neighborhood $U$ such that the associated $\mathcal{R}: p^{-1}(U) \rightarrow p^{-1}(x)$ restricts to an isomorphism $\left(\mathcal{R} \mid p^{-1}(y)\right)_{*}$ : $H_{n}\left(p^{-1}(y)\right) \rightarrow H_{n}\left(p^{-1}(x)\right)$ for all $y \in U$. Establishing that $B$ equals the continuity set of $p$ is a cornerstone for showing an $N$-like map $p$ is an approximate fibration.

The following is basic for investigating codimension- $k$ PL fibrators. 
Proposition 2.1. [5, Theorem 5.4] Suppose that $p: M^{n+k} \rightarrow B$ is an approximate fibration such that each $p^{-1}(b), b \in B$, collapses to an $n$-complex homotopy equivalent to a closed $n$-manifold. Then $B$ is a simplicial homotopy $k$-manifold (topological $k$-manifold).

Proposition 2.2. [5, Proposition 2.1] Let $p: M^{n+k} \rightarrow \mathbb{R}^{k}$ be codimension- $k$ map from an orientable $(n+k)$-manifold $M^{n+k}$ onto Euclidian $k$-space such that each fiber is homotopy equivalent to an orientable $n$ manifold $N$. Suppose that $p$ is an approximate fibration over $\mathbb{R}^{k} \backslash \mathbf{0}$. Then $p$ has Property $\mathcal{R}_{i}^{\cong}$ for all $i<k-1$. Furthermore, if $p$ has Property $\mathcal{R} \cong$ $\cong$, then for all $y \in \mathbb{R}^{k} \backslash \mathbf{0}$, the degree of map $\mathcal{R} \mid p^{-1}(y): p^{-1}(y) \rightarrow$ $p^{-1}(\mathbf{0})$ is one.

\section{Codimension $k$ fibrators}

In this section, we show that some manifolds satisfy necessary and sufficient conditions to be codimension- $k$ PL fibrator.

Lemma 3.1. [14] Suppose $N$ is a closed $n$-manifold with residually finite, torsion free $\pi_{1}(N)$ and finite $H_{1}(N)$. Then $N$ is a codimension-2 fibrator if and only if $N$ does not cover itself regularly and cyclically, up to homotopy type, provided either $\pi_{k}(N) \cong 0$ for $1<k<n-1$ or $n \leq 4$.

Theorem 3.2. [15, Theorem 3.5] Suppose $N$ is a closed, hopfian PL manifold which is both $\pi_{k}(N)=0$ for $1<k<n-1$ and a codimension-2 $P L$ fibrator and whose fundamental group has Property NCSA. Then $N$ is a codimension- $(n-1) P L$ fibrator.

TheOREM 3.3. Suppose $N$ is a closed PL $n$-manifold, codimension-2 fibrator, $\pi_{k}(N)=0$ for $1<k<n-1, \pi_{n-1}(N)$ finite and $\pi_{1}(N)$ has Property NCSA. Then $N$ is a codimension-n PL fibrator.

Proof. It is known that $N$ is a codimension- $(n-1)$ PL fibrator by Theorem 3.2. Suppose that $p: M \rightarrow B$ is an $N$-like PL map defined on an orientable $2 n$-manifold. First, we will show that $B$ is an $n$-manifold. Let $L$ denote a link about an arbitrary vertex $v \in B$ and $S$ the star of $v$ in the same implicit triangulation. Set $L^{\prime}=p^{-1}(L)$ and $S^{\prime}=p^{-1}(S)$. Since $N$ is a codimension- $(n-1)$ PL fibrator, $p \mid L^{\prime}: L^{\prime} \rightarrow L$ is an approximate fibration, implying that $L$ is an $(n-1)$-manifold by Proposition 2.1. In the homotopy exact sequence for $p \mid L^{\prime}$,

$$
\cdots \rightarrow \pi_{k}(N) \rightarrow \pi_{k}\left(L^{\prime}\right) \rightarrow \pi_{k}(L) \rightarrow \pi_{k-1}(N) \rightarrow \cdots
$$


we have $\pi_{k}\left(L^{\prime}\right) \cong \pi_{k}\left(S^{\prime}\right) \cong \pi_{k}(N)$ for $k \leq n-2$ by general position for the first isomorphism and by the fact that $p^{-1}(v)$ is a strong deformation retract of $S^{\prime}$. Since $\pi_{k}(N)=0$ for $1<k<n-1$ and $\pi_{1}(N)$ has Property NCSA, we have $\pi_{k}(L)=0$ for $1<k<n-1$, implying that $L$ is a homotopy sphere and $B$ is a manifold by Proposition 2.1.

Case I: $\pi_{1}(N)$ is finite; then $N$ is a codimension- $n$ PL fibrator [1, Theorem 7.4].

Case II: $\pi_{1}(N)$ is infinite; consider the universal covering spaces $\widetilde{S}^{\prime}$ of $S^{\prime}$ and $\widetilde{N}$ of $N$, and let $i: p^{-1}(w)(\simeq N) \rightarrow S^{\prime}$ be the inclusion for all $w \in S$. Since $p \mid L^{\prime}: L^{\prime} \rightarrow L$ is an approximate fibration and $\pi_{1}(N)$ has Property NCSA, from the homotopy exact sequence for $p \mid L^{\prime}$

$$
\cdots \rightarrow \pi_{2}(L) \rightarrow \pi_{1}(N) \rightarrow \pi_{1}\left(L^{\prime}\right) \rightarrow \pi_{1}(L) \rightarrow 1
$$

$q \circ R: p^{-1}(w) \rightarrow p^{-1}(v)$ induces a $\pi_{1}$-isomorphism, where $R: S^{\prime} \rightarrow$ $p^{-1}(v)$. By appealing to the method of the proof in [16, Lemma 3.1], we see that for all $w \in S, q^{-1}\left(p^{-1}(w)\right)$ is connected and has the homotopy type of $\tilde{N}$. From the following diagram

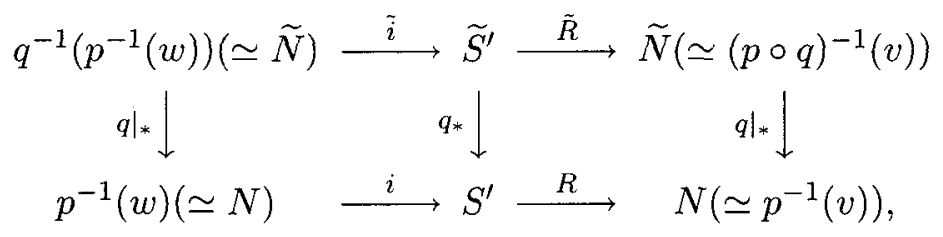

we see $\operatorname{deg} R \circ i=\operatorname{deg} \widetilde{R} \circ \tilde{i}$, where $\left.\tilde{R}: \widetilde{S}^{\prime} \rightarrow(p \circ q)^{-1}(v)\right)$ is the lifting of $R: S^{\prime} \rightarrow p^{-1}(v)$. This diagram is a pull back diagram, so it is easy to see that $\tilde{R} \circ \tilde{i}: \tilde{N} \rightarrow \widetilde{S}^{\prime}$ is a proper map. Since $\pi_{1}(N)$ has Property NCSA and $\pi_{k}(N)=0$ for $1<k<n-1, R \circ i$ induces a $H_{k}$-isomorphism for $1 \leq k<n-1$ [7]. Finiteness of $\pi_{n-1}(N)$ implies that $H_{n-1}(\widetilde{N}) \cong \pi_{n-1}(\widetilde{N})$ is finite by the Hurewicz theorem. Consider the following diagram of exact sequences [21, Lemma 1.1],

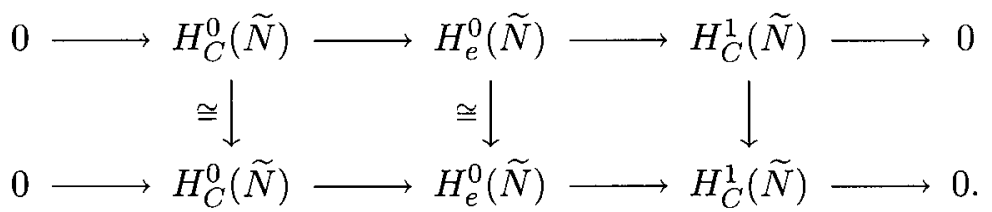

Here all the vertical maps are induced by $\tilde{R} \circ \tilde{i}$ and $H_{e}^{0}$ denotes the space of ends. Since the first and second maps are isomorphisms, the third map is an isomorphism by the Five Lemma. From the Poincare duality theorem, we have the following diagram 


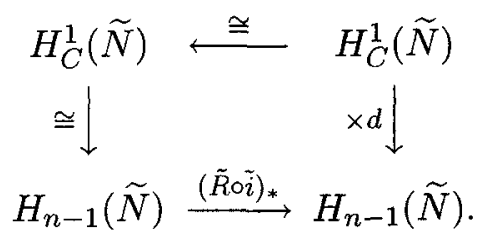

Here the horizontal isomorphism is induced by $\tilde{R} \circ \tilde{i}$. Hence, we see $\left[H_{n-1}(\widetilde{N}):(\tilde{R} \circ \tilde{i})_{*}\left(H_{n-1}(\widetilde{N})\right)\right]=\operatorname{deg} \widetilde{R} \circ \widetilde{i}=d$. Since $p \circ q \mid: \widetilde{L}^{\prime} \rightarrow L$ is an approximate fibration with fiber $\widetilde{N}$ and $L$ is a sphere, we have the following Wang's exact sequence

$$
\begin{aligned}
& (0 \cong) H_{1}(\tilde{N}) \rightarrow H_{n-1}(\tilde{N}) \rightarrow H_{n-1}\left(\tilde{L}^{\prime}\right) \\
& \rightarrow H_{0}(\tilde{N})(\cong Z) \rightarrow H_{n-2}(\tilde{N})(\cong 0) \rightarrow \cdots .
\end{aligned}
$$

From the homology exact sequence of $\left(S^{\prime}, L^{\prime}\right)$ and the simple diagram chasing, we have an isomorphism $(\widetilde{R} \circ \widetilde{i})_{*}: H_{n-1}(\widetilde{N}) \rightarrow H_{n-1}\left(\widetilde{S}^{\prime}\right)$, and so $\operatorname{deg} R \circ i=\operatorname{deg} \widetilde{R} \circ \widetilde{i}=1$ by Proposition 2.2. By Swarup [21, Lemma 1.1], $N$ is a hopfian manifold. Hence, $R \circ i: N \rightarrow S^{\prime}$ is a homotopy equivalence and $p$ is an approximate fibration.

REMARK. Finiteness of $\pi_{n-1}(N)$ in Theorem 3.3 cannot be omitted since $S^{n-1} \times S^{1}$ fails to be a codimension- $n$ PL fibrator.

COROLlary 3.4. Suppose $N$ is a closed $n$-manifold with residually finite, torsion free $\pi_{1}(N)$, finite $H_{1}(N)$ and $\pi_{k}(N) \cong 0$ for $1<k<n-1$. Then $N$ is a codimension-n fibrator if and only if $N$ does not cover itself regularly and cyclically up to homotopy type, provided $\pi_{1}(N)$ has Property NCSA.

Proof. The result follows from Lemma 3.1 and Theorem 3.3.

COROLlary 3.5. Suppose $N$ is a closed aspherical $n$-manifold with residually finite and $\chi(N) \neq 0$. Then $N$ is a $P L$ fibrator

Proof. It is known that $N$ is a codimension-2 fibrator [5]. Since $N$ is an aspherical manifold with $\chi(N) \neq 0, \pi_{1}(N)$ has the property NCSA [19]. The result follows from Theorem 3.3 .

Theorem 3.6. Suppose $N$ is a closed 3-manifold with residually finite, torsion free $\pi_{1}(N)$, finite $H_{1}(N)$. Then $N$ is a codimension-3 fibrator if and only if $N$ does not cover itself regularly and cyclically up to homotopy type, provided $\pi_{1}(N)$ has Property NCSA. 
Proof. It is known that every closed 3-manifold is hopfian [11], and $H_{2}(N) \cong H^{1}(N) \cong 0$ by the Poincare duality theorem and the universal coefficient theorem. Apply Proposition 2.2 and Lemma 3.1.

THEOREM 3.7. Suppose $N$ is a closed 4-manifold with residually finite, torsion free $\pi_{1}(N)$, finite $H_{1}(N)$. Then $N$ is a codimension-4 fibrator if and only if $N$ does not cover itself regularly and cyclically up to homotopy type, provided $\pi_{1}(N)$ has Property NCSA and $\beta_{2}(N)>2$.

Proof. Finiteness of $H_{1}(N)$ implies $\chi(N) \neq 0$ and $H_{3}(N) \cong H^{1}(N)$ $\cong 0$. Having confirmed that every closed 4-manifold is hopfian [11], apply Proposition 2.2, Lemma 3.1 and [10, Proposition 4.1].

\section{References}

[1] N. Chinen, Finite groups and approximate fibrations, Topology Appl. 102 (2000), $59-88$.

[2] D. S. Coram and P. F. Duvall, Approximate fibration, Rocky Mountain J. Math. 7 (1977), 275-288.

[3], Approximate fibration and a movability condition for maps, Pacific J. Math. 72 (1977), 41-56.

[4] - Mappins from $S^{3}$ to $S^{2}$ whose point inverses have the shape of a circle, General Topology Appl. 10 (1979), 239-246.

[5] R. J. Daverman, Submanifold decompositions that induce approximate fibrations, Topology Appl. 33 (1989), 173-184.

[6] , Hyperhopfian groups and approximate fibrations, Compositio Math. 86 (1993), 159-176.

[7] , The PL fibrators among aspherical geometric 3-manifolds, Michigan Math. J. 41 (1994), 571-585.

[8] — 180-192.

[9] , Manifolds that induce approximate fibrations in the PL category, Topology Appl. 66 (1995), 267-297.

[10] R. J. Daverman, Y. H. Im and Y. Kim, Connected sums of 4-manifolds as codimension- $k$ fibrators, submitted.

[11] J. C. Hausmann, Geometric hopfian and non-hopfian situations, Lecture Notes in Pure Appl. Math. Marcel Decker, Inc., NY (1987), 157-166.

[12] Y. H. Im and Y. Kim, Hopfian and strongly hopfian manifolds, Fund. Math. 159 (1999), 127-134.

[13] , Necessary and sufficient conditions for s-Hopfian manifolds to be codimension-2 fibrators, Proc. Amer. Math. Soc. 129 (2001), no. 7, 2135-2140.

[14] _ Manifolds that fails to be codimension-2 fibrators necessarily cover themselves, J. Austral. Math. Soc., to appear.

[15] Y. H. Im and S. H. Kim, Approximate fibrations on PL manifolds, Bull. Korean Math. Soc. 35 (1998), no. 3, 491--501.

[16] Y. Kim, Strongly Hopfian manifolds as codimension-2 fibrators, Topology Appl. 92 (1999), 237-245. 
[17] _ Manifolds with hyperhopfian fundamental group as codimension-2 fibrators, Topology Appl. 96 (1999), 241-248.

[18] J. R. Munkres, Elements of algebraic topology, Addison-Wesley, Menlo Park, Calif., 1984.

[19] S. Rosset, A vanishing theorem for Euler characteristics, Topology Appl. 185 (1984), 211-215.

[20] E. H. Spanier, Algebraic topology, McGraw Hill, 1966, Corrected reprint of the 1966 original, Springer-Verlag, 1995.

[21] G. A. Swarup, On a theorem of C. B. Thomas, J. London Math. Soc. 8 (1974), $13-21$.

Department of Mathematics

Pusan National University

Pusan 609-735, Korea

E-mail: yhim@pusan.ac.kr 